\title{
EMERGING SCIENCE AND RESEARCH OPPORTUNITIES FOR METALS AND METALLIC NANOSTRUCTURES: A REPORT ON THE NSF MMN WORKSHOP
}

\author{
Tresa Pollock ${ }^{1}$, Carol Handwerker ${ }^{2}$ \\ ${ }^{1}$ University of California Santa Barbara; Materials Department; Santa Barbara, CA; 93106-5050; USA \\ ${ }^{2}$ Purdue University; 701 W. Stadium Avenue; West Lafayette, IN; 47907; USA
}

\section{Extended Abstract}

In the next decade, fundamental research in metals and metallic nanostructures (MMN) has the potential to continue to transform science into innovative materials, devices, and systems. This talk summarizes the findings of a workshop to identify emerging and potentially transformative research areas in MMN. The metals and metallic nanostructures (MMNs) workshop aimed to identify significant research trends, scientific fundamentals, and recent breakthroughs that can enable new or enhanced MMN performance, either alone or in a more complex materials system, for a wide range of applications. Additionally, the role that MMN research can play in high-priority research and development (R\&D) areas such as the U.S. Materials Genome Initiative, the National Nanotechnology Initiative, the Advanced Manufacturing Initiative, and other similar initiatives that exist internationally was assessed. The workshop also addressed critical issues related to materials research instrumentation and the cyberinfrastructure for materials science research and education, as well as science, technology, engineering, and mathematics (STEM) workforce development, with emphasis on the United States but with an appreciation that similar challenges and opportunities for the materials community exist internationally.

The societal and economic impacts of MMN research will continue to be significant. Metals and metallic nanostructures underlie systems and technologies critical to solving national and global grand challenges. Innovations in metals, metal alloys, and metallic nanostructures are needed to advance systems for:

- energy generation, harvesting and storage thermoelectrics, batteries, fuel cells, advanced turbines, magnetic induction, motors, hydrogen production and storage, nuclear reactor materials, separation membranes

- propulsion and transportation - high strength, high performance lightweight structures, materials designed for improved creep, impact, fatigue or corrosion resistance, high temperature metals, alloys and coatings for extreme environments, non-destructive evaluation

- electronics, telecommunication, and information technology - electronic packaging, process modeling, device nanostructures to overcome the end to Moore's law, thin film and nanostructured electrical interconnects, magnetic devices and motors, and sensors

- sustainability - nanostructured metals for catalytic convertors, polymer catalysis, hydrogen and ammonia production, low pollution smelting and refining processes

- health care - imaging systems, integrated in-vivo sensors, electronics, and communication systems for health monitoring and drug delivery, prosthetics and implant materials and systems, cancer treatment technologies
- manufacturing - additive and nano-manufacturing technologies, non-equilibrium processing approaches, recycling and reuse, rapid deployment of alternatives to "critical" materials.

A central theme of the Workshop was the view that research in metals and metallic nanostructures has provided and will continue to provide benefit to the nation through the coupling of experiment, theory, and simulation to link atomistic, nanoscale, microscale, and mesoscale phenomena across time scales for an ever widening range of applications. Within this overarching theme, the workshop participants identified emerging research opportunities that are categorized in terms of:

- 3-D and 4-D Materials Science

- Structure Evolution and the Challenge of Heterogeneous and Multicomponent Systems

- The Science Base for Property Prediction Across the Lengthscales

- Nanoscale Phenomena at Surfaces - Experiment, Theory, Simulation

- Prediction and Control of the Morphology, Microstructure, and Properties of "Bulk" Nanostructured Metals

- Functionality and Control of Materials Far from Equilibrium

- Hybrid and Multifunctional Materials Assemblies

- Materials Discovery and Design: Enhancing the Theory Simulation - Experiment Loop

This talk will summarize the findings of the workshop and will provide a brief overview of these emerging research opportunities, along with challenges and opportunities for the materials community in the areas of instrumentation, cyberinfrastructure, education, and workforce development. 